\title{
BRAIN METASTASES
}

Brain metastases develop when cells from tumours - most commonly breast, lung and colorectal cancers, renal cell carcinoma and melanoma - spread to the brain. Malignant spread to the brain is associated with extremely poor outcomes.

\section{EPIDEMIOLOGY}

An estimated $20 \%$ of all patients with cancer will develop brain metastases, with advancedstage and aggressive cancers increasing the risk. Combined with primary tumour type and subtype, factors such as age might influence the brain metastasis risk; for example, younger patients (20-39 years of age) with breast cance have the highest risk for intracranial spread, whereas melanoma is more likely to produce brain metastases in patients aged $50-59$ years.

\section{DIAGNOSIS}

Brain metastases should be suspected in any patient with known systemic cancer in whom neurological findings, such as headache, weakness,

develop; imaging confirmation can be sufficient to prompt treatment. However, in patients without a known (or with a previously undetected) primary tumour, or in whom a previous cancer was eradicated some time ago, identifying the source and ruling out a primary brain tumour is essential. Neuroimaging, histopathology and genetic expression profiles can all be used to determine the composition of the tumour.

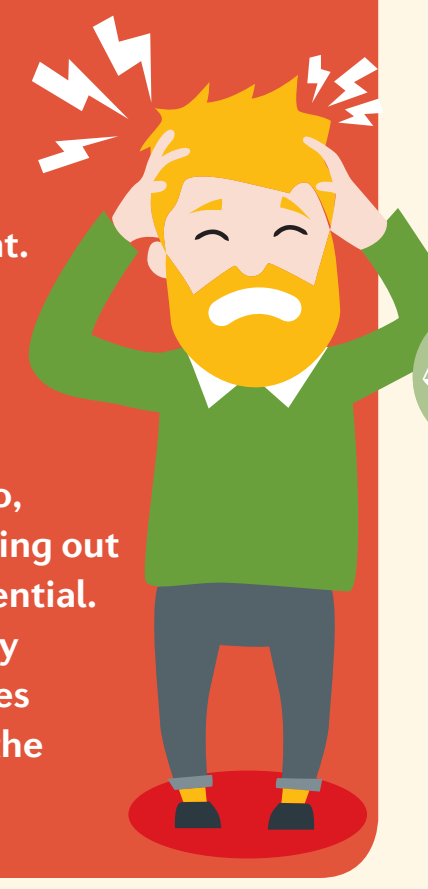

MANAGEMENT

\section{- - - - - - - - - -}

Current

treatment of

brain metastases

typically includes

a combination

of surgery,

radiotherapy

and/or systemic

medical therapies.

'..... T-....

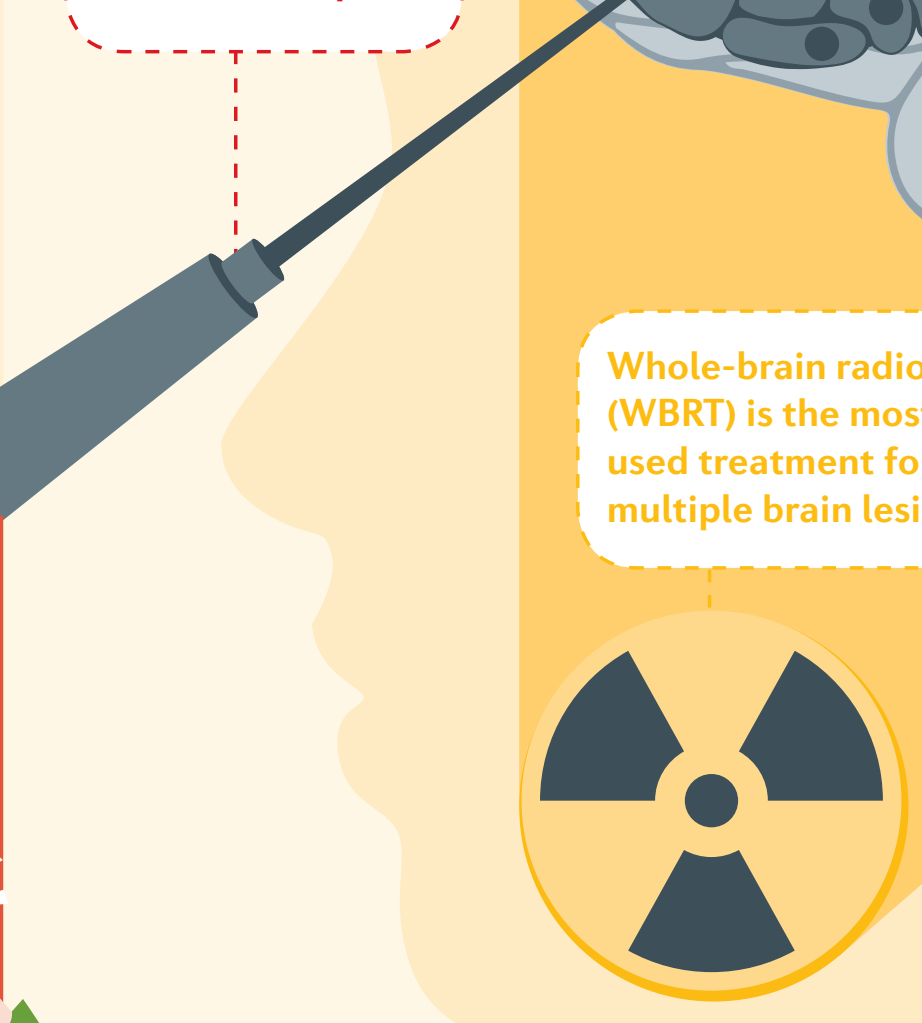

QUALITY OF LIFE

Patients with brain metastases have significantly reduced quality of life owing to the presence of of neurological deficits that interrupt routine activities, and the adverse effects associated with aggressive treatments. For example, WBRT negatively affects physical or cognitive function and is associated with fatigue, appetite loss, drowsiness, hair loss and weakness; SRS is superior to WBRT in term of neurocognitive effects.
For the Primer, visit doi:10.1038/s41572-018-0055-y

\section{MECHANISMS}

To form a brain metastasis, tumour cells break away from the primary tumour and invade the surrounding tissues, blood vessels and the lymphatic system. Once in the circulation, tumour cells begin the process of metastatic extravasation from the vasculature, which is facilitated by these cells undergoing adhesive (circulatory) arrest. Brain metastases tend to occur at the border between grey and white matter and areas between vascular territories, as longer relative mean transit of blood flow provides more time for the cells to overcome the blood-brain barrier and egress from the vasculature. Brain colonization involves astrocytes, microglia, complex signalling and tumour cell predisposition or adaptation, among other factors,

to be successful.

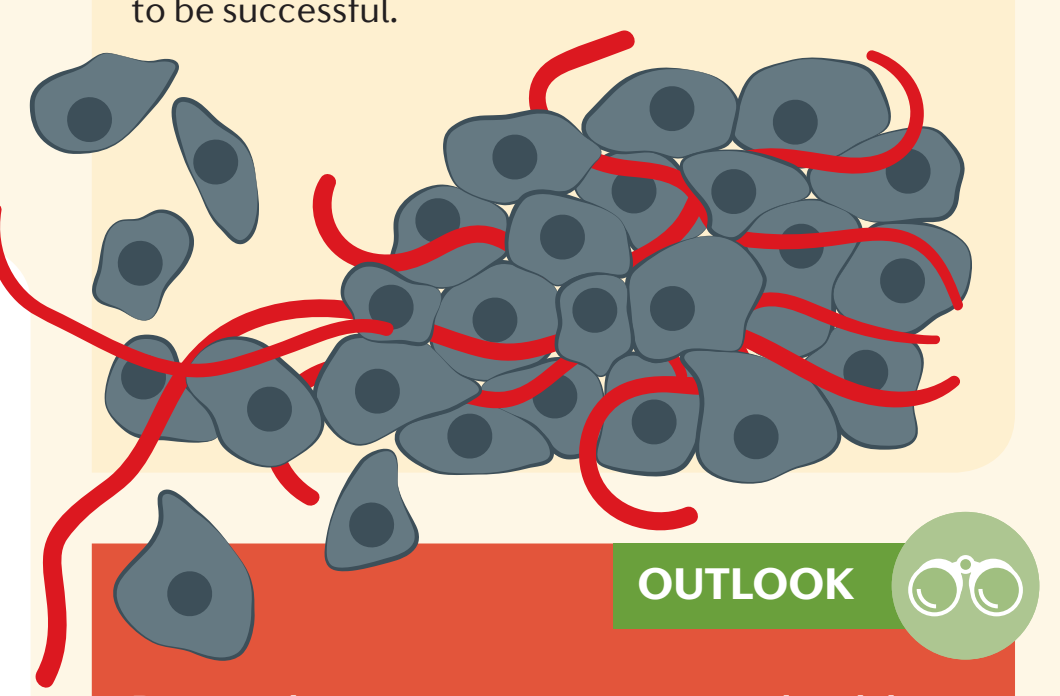

Despite the poor prognosis associated with brain metastases, as our fundamental understanding

of the biology of this disease increases, so too will our ability to treat them effectively. Strategies being pursued include addressing the low and heterogeneous permeability of brain metastases to therapeutic agents (such as targeted drug carrier systems), targeting various steps in metastatic cell colonization and early tumour growth, and developing methods to safely obtain specimens in a minimally invasive manner to better understand the genetics of brain metastases over time. 